Corps et culture

Numéro 6/7 | 2004

Métissages

\title{
Le temps métissé des joutes languedociennes : un « entre deux » révélateur
}

Jérôme Pruneau

\section{(2) OpenEdition}

1 Journals

Édition électronique

URL : http://journals.openedition.org/corpsetculture/816

DOI : 10.4000/corpsetculture.816

ISSN : $1777-5337$

Éditeur

Association Corps et Culture

Édition imprimée

Date de publication : 1 janvier 2004

ISSN : 1268-5631

Référence électronique

Jérôme Pruneau, "Le temps métissé des joutes languedociennes : un "entre deux » révélateur », Corps et culture [En ligne], Numéro 6/7 | 2004, mis en ligne le 11 octobre 2007, consulté le 08 septembre 2020. URL : http://journals.openedition.org/corpsetculture/816 ; DOI : https://doi.org/ 10.4000/corpsetculture.816

Ce document a été généré automatiquement le 8 septembre 2020

(c) tous droits réservés 


\title{
Le temps métissé des joutes languedociennes : un « entre deux » révélateur
}

\author{
Jérôme Pruneau
}

1 L'article qui suit est un questionnement, une réflexion qui fait suite à un travail de thèse sur les joutes languedociennes (Pruneau J., 2001) dans lequel les notions « d'entre deux », de "métissage ", "d'hybridation », de "médiation » ou de "compromis » participent à la relecture des jeux traditionnels ou des sports modernes, en considérant qu'il est possible de sortir des schèmes classiques, des taxinomies imposantes, des classifications réductrices ou des nomenclatures d'opposition par la voie du «ternaire ». En proposant l'oxymore de «sport traditionnel » dans le titre même de la thèse (Pruneau J., 2001), il s'agit de déstabiliser l'ordre établi envers une classification duale des jeux et des sports - déconstruire le rapport entre tradition et modernité dans son sens habituellement admis et opposé - et montrer, à travers les joutes, qu'une solution distincte $d u$ «binaire » peut non seulement émerger, mais s'inscrire dans la lecture du social tel que celui-ci est travaillé aujourd'hui. Ce qui renvoie immédiatement à des questions phares : qu'entend-on par ces aspects de métissage? Pourquoi ces notions font-elles écho aujourd'hui dans d'autres sphères que l'interculturel qui en est le berceau? Que faut-il y déceler pour comprendre le monde social et le champ du sport en particulier? Quelles perspectives propose cette lecture de «l'entre deux »?

2 Autant de questions auxquelles la présente et courte contribution n'a pas la prétention de répondre mais dont la finalité vise davantage à poser les bases d'une réflexion de fond. Sortir du «tout blanc » ou du « tout noir » dans lesquels sont inscrits les valeurs morales de nos sociétés (bien/mal), les schémas éducatifs (scolarisé/marginalisé), les idéologies dominantes (droite/gauche), doit être une réponse aux interstices grandissants du monde social, siège de «l'entre deux ». Vivre et accepter d'être dans le « gris » en composant avec les interactions des systèmes qui se rencontrent - et non les oppositions systématiques -, n'est-ce pas là un état d'esprit en adéquation avec le réel ? 
Réel vécu de l'hypermodernité aux accélérations si fulgurantes que le passé - l'hier est immédiatement relégué au rang du "has been" avec lequel on ne sait plus conjuguer. C'est un point de départ possible du questionnement, comme une équation mathématique dont les variables se prêtent au jeu de la commutativité : [gestion d'un héritage + gestion du devenir] ? A n'en pas douter, se pencher sur le problème est un début de solution. C'est ici l'essai proposé à travers l'exemple des joutes dont l'observation offre un terrain propice à une amorce de réponse.

Le « compromis » des joutes languedociennes : une solution pour exister, un autre schéma de société

3 Les formes de contact entre deux systèmes culturels sont traitées dans l'anthropologie sociale à travers divers schémas explicatifs: l'acculturation, la transculturation ou néoculturation (Herskovitz M-J., 1958 ; Balandier G., 1971, 1974 ; Bastide R.,1967, 1970, 1971; Augé M., 1975), la créolisation (Chaudenson R., 1979), le métissage ou l'hybridation (Amselle J-L., 1990, Audinet J., 1999), sont autant de concepts qui tentent de repérer les modes opératoires de ces échanges où se jouent la complexité, la diversité et la singularité des cultures dans un contexte d'uniformisation qui s'abreuve à la source d'une modernité globalisante. Toutes ces différentes typologies s'emploient à désigner un changement culturel dans lequel des groupes sociaux subissent ou se voient imposer des traits culturels d'une autre société. Les nuances qui apparaissent entre ces termes montrent combien il est difficile de proposer un modèle de processus qui opérerait de façon universelle dans les formes de rencontres entre les sociétés. Pour autant, devant la complexité à établir une loi générale, des tendances récurrentes émergent et signalent un consensus des auteurs. Dans ces configurations, «la société industrielle avancée condamne à l'inefficacité les formes traditionnelles de solidarité qui s'exprimaient à travers les communautés régionales et locales » (Abou S., $1981: 18$ ). Face à cet univers de transformations, les populations mettent en place des formes de réaction liées à l'instinct de conservation, «en cherchant des formules de remplacements susceptibles de leur restituer des liens de solidarité organique et des médiations culturelles efficaces » (ibid: 19).

4 Par analogie, l'entrée dans la modernité du système culturel des joutes par le truchement de la "sportivisation» (1945) signe un contact culturel déstabilisant au regard d'un équilibre structurel séculaire conquis à l'échelle du temps (les joutes existent à Sète depuis 1666). Cependant, ce ne sont pas ici deux groupes ethniques qui se mélangent mais davantage deux systèmes de valeurs qui entrent en confrontation. D'un côté, la structure transhistorique des joutes ${ }^{1}$ est garante d'une continuité de la forme traditionnelle de pratique autour d'un fonctionnement communautaire. De l'autre, la figure d'une modernité sportive est porteuse de tensions et de tourmentes à travers les nouvelles donnes du système fédéral qui tendent à subordonner les différents aspects de la culture traditionnelle aux modèles de comportement et de pensée inhérents à la compétition et à l'individualisme. Au carrefour, là où les processus d'acculturation liés à la collision culturelle amènent à l'assimilation ou à la différenciation, une dialectique se met en place pour concilier - et non opposer tradition et modernité. Elle s'entend au sens de Balandier, dans son mode opératoire, comme ce qui permet « de saisir l'hétérogénéité et de parvenir à la compréhension de ce qu'il y a de dynamique en tout phénomène » (Balandier G., 1971 : 73). En cela, « toute réalité suppose un compromis instable, variable, difficile à saisir, entre continuité et discontinuité » (Gurvitch G., 1968, 70). 
Ce compromis ou plutôt cette médiation, c'est la solution de l'équation posée ci-dessus. Entre perpétuation et renouvellement, entre gestion d'un héritage et celle d'un devenir, une régulation s'opère pour contrôler les tensions naissantes et menaçantes dans les joutes. En combinant les deux registres de valeurs, les personnes du même système culturel global parviennent à conserver une lisibilité traditionnelle et communautaire, du moins à laisser transparaître l'image d'une identité voulue comme telle. Au final, une forme hybride de pratique tient lieu de résultante, en assortissant des éléments de traditions avec des substrats typiquement caractérisés par le sport, mieux encore par le spectacle sportif. Le processus de compromis opère comme une réponse dynamique à des tensions nées des aspects de modernité, symbolisée par la «sportivisation». Celle-ci, à la fois vecteur du développement de la pratique par la multiplication des sociétés, des tournois et des jouteurs qu'elle engendre, à la fois porteuse de contraintes et d'un étiolement des valeurs communautaires, uniformise l'activité au diapason d'une réalité sportive normative. Pour pallier cette dérive axiologique, la médiation apparaît comme l'intégration de ce modernisme globalisant sous des physionomies traditionnelles qui n'en sont plus forcément, du moins qui profitent d'une injonction de la contemporanéité pour se revigorer et s'affirmer davantage, voire émerger tels de beaux semblants au bénéfice d'une lisibilité souhaitée. De sorte que, pour assurer la continuité de l'authenticité originelle de leur pratique sans s'enfermer dans un repli identitaire sclérosant, les acteurs des joutes ne sont pas prédisposés à renoncer aux avantages d'une société technologique ou techniciste. Pour autant, il ne s'agit pas non plus d'être phagocyté ou d'en subir les effets d'assimilation ou d'acculturation au risque d'un effacement des principes fondamentaux incarnés par les propriétés structurelles. Simplement, la culture traditionnelle des joutes potentialise l'universalité qu'elle porte en elle sous la forme d'une mise en conformité sportive, afin de préserver une figure locale d'authenticité. Vivante, en mouvement, elle « change à partir de son patrimoine assumé et réinterprété et garde un profil qui lui est particulier »(Abou S., 1981:14).

6 En cela, un troisième espace s'ouvre, sorte d'interstice entre tradition et modernité, dans lequel la dialectique à l'œuvre permet de concilier un patrimoine culturel avec une ouverture au monde, non pas en terme de diffusion extra territoriale comme dans la pelote basque par exemple, mais en terme de dynamique locale qui renforce une visibilité identitaire territoriale. Cet intervalle prend alors la figure d'une concordance muette dont on se sert des bienfaits qu'elle produit à des fins identitaires. Mieux encore, un syncrétisme axiologique s'opère sous la forme d'une alchimie de deux systèmes de valeurs qu'il serait naturellement commode d'opposer selon une vision binaire ou dualiste prédominante dans nos systèmes de pensée. Les deux pôles, tradition et modernité, ne sont pas ici figés dans une opposition où le premier résiste à l'autre dans une forme de foyer circonscrit, replié parce qu'en proie aux agressions, tel un village d'irrésistibles gaulois qui refuse un avenir romain moderniste. Non plus, d'ailleurs, positionnés sur une échelle de temps linéaire où le second, emprunt de raison individuelle, de scientisme et de rationalité, succède naturellement au premier, siège d'une existence communautaire, en l'annulant, l'effaçant ou l'englobant automatiquement.

7 En fait, dans l'écart de ces pôles, les acteurs des joutes, à la recherche d'un modèle viable de liens, de relations, d'échanges qui permet l'expression des deux systèmes de valeurs, produisent sur un mode ternaire une médiation entre les tensions que la 
rencontre de ces pôles procure. En se nourrissant mutuellement, les aspects de tradition et de modernité ouvrent ici un espace non recensé dans les pratiques sportives. Là où la «sportivisation » a phagocyté l'identité culturelle de certains jeux traditionnels par un effet de diffusion globalisant et uniformisant, les joutes ont su conserver localement une lisibilité d'authenticité traditionnelle, au regard de la modernité. Le compromis qui accompagne cette réussite intervient comme le processus qui permet d'éviter une fracture irréversible. Dans cette configuration, la pratique à mi-chemin entre culture traditionnelle, sport et spectacle, se conjugue sur un caractère hybride où les rites traditionnels se transforment mais assurent ses fondements, les enjeux sportifs lui garantissent son renouvellement et le spectacle sa pérennité. Sans doute est-ce pour cela qu'à la question posée à un jouteur sur la définition de sa pratique, la réponse fut : « les joutes, c'est différent des autres sports, c'est autre chose, c'est entre du sport et des traditions, voilà ».

8 En définitive, cet exemple traité à travers le système des joutes, c'est l'ouverture au monde sur un registre ternaire où la dimension de conciliation prend le relais d'une confrontation ailleurs conjuguée sur le mode de la dualité. A l'opposition usuellement orchestrée se substitue un espace hybride, source d'une possible articulation entre tradition et modernité. Les joutes sont un «sport traditionnel » entre spectacle et rituel, entre performance et jeu, entre compétition et loisir, entre activité touristique locale et patrimoine culturel. Elles donnent aujourd'hui toute la mesure d'une réussite culturelle qui s'entend comme une "authenticité anachronique ». Là où les autres pratiques traditionnelles n'ont pas su négocier l'avènement de la «sportivisation » en s'accommodant de ses injonctions, parce qu'elles s'y sont soumises totalement ou parce qu'elles l'ont refusé, les acteurs des joutes sont parvenus localement à l'orienter favorablement, sorte d'accord, d'entente métissée. En cela, le compromis repose sur le postulat qu'il est admissible de faire agir ensemble des valeurs issues de systèmes classiquement opposés afin d'élaborer un syncrétisme axiologique. Celui-ci joue le rôle de révélateur, dans un espace en mouvement, entre les expériences du passé encore à l'œuvre et les inventions nées de la contemporanéité. C'est un espace ouvert où rien ne semble être établi, fixé ou figé en son centre, si ce n'est un champ de possibles imprévisibles, initialement inconcevable par la Doxa et, par ailleurs, contingent. Le compromis est une forme de "tiers", qui autorise la communication, telle une conjoncture en création qui s'élabore par rapport à deux pôles distincts, lesquels s'y reconnaîtront tout en acceptant la nouveauté. Ici, il n'y a pas de «dualité mortelle qui enferme » (Audinet J., 1999 : 107) mais simplement un « entre deux » qui, tout autant qu'il se glisse entre les repères usités, s'en construit de nouveaux qui lui sont propres. En fait, la médiation permet un desserrement des schèmes classiques de pensée en proposant l'ouverture, certes, incertaine, mais qui rompt avec l'harmonie ancienne où se cristallise le monde symbolique habituellement admis (bien/mal, blanc/noir, global/ local, universel/particulier, tradition/modernité, rites/religion, sacré/profane, etc.). En cela, les classifications achoppent devant la béance qui s'ouvre comme une dynamique, non un état figé. Quand dans l'opposition, l'un et l'autre des pôles ne concorderont jamais, de leurs tensions émergentes peut naître le compromis en mouvement, siège d'un syncrétisme opératoire.

Le champ sportif dans les grilles du " gris »?

9 Si cette lecture, construction plausible d'un interstice non révélé dans le champ des pratiques physiques traditionnelles est recevable, est-il possible alors d'élargir son champ d'application? Par exemple, l'élaboration de cet espace de médiation n'a-t-il pas 
un rôle à jouer dans la perception et la compréhension du sport tel qu'il est étiré, tiraillé et finalement complexifié aujourd'hui ? Ce cadre intelligible n'offre-t-il pas, en s'y référant, un éclairage nouveau sur les enjeux de l'hétérogénéité sportive?

10 La sociologie des pratiques physiques a clairement identifié les phases axiologiques du développement du sport depuis les années 1960, en considérant notamment différentes phases homogènes: l'émergence des loisirs annoncée par Joffre Dumazedier (1962); l'éloge de l'individualisme en quête d'hédonisme hic et nunc dans les années 1970, source de la diversification des pratiques; puis la redécouverte des corps en prise au mythe de l'éternelle jeunesse dans la décennie suivante; la consommation sportive, enfin, dans les années 1990 où, à l'image d'une société hyper-modernisée, le sport "s'hyper-consomme » dans toutes ses modalités, sous toutes ses formes, dans tous ses espaces (effet zapping). Cette "sur-sportivisation" qui surgit, aliénée à l'ordre entrepreneurial des marques, des enseignes, des sponsors et du marketing au point que les «sportifs contemporains [seraient] de plus en plus sensibles aux exhortations de certaines marques » (Loret A., 1996) ne tend-elle pas à brouiller les cartes d'une vision jusqu'alors perceptible? Du moins, soumise aux idéologies classiques qui enferment le "Sport» dans une représentation dichotomique, sport de compétition et sport de loisirs, sport professionnel et sport amateur, sport institutionnalisé et sport «fun » et dans lesquelles, comme le suggère Martine Segalen, «d'une part, on aurait le sérieux, l'argent, la compétition, l'effort [...] et d'autre part, le plaisir, la gratuité relative, la participation et la convivialité » (Segalen M., $1994: 231$ ). Que ce soit sous les grilles interprétatives de «la distinction» (Bourdieu P., 1979), de la "post-modernité » (Maffesoli M., 1996) ou de la "contre culture » (Loret) ${ }^{2}$, les auteurs sont formels, la société sportive est en rupture avec ces conceptions et les nouvelles formes de pratique recensées mettent à mal les institutions. Pour autant, que l'on parle des jeunes « skateboarders ", des « riders » en roller ou en B. M. X ou des « kite-boarders » en fly-surf, l'analyse se fait plus confuse et exprime in fine soit une résistance au changement, soit une rupture en terme d'imaginaire social, soit une opposition à la culture sportive qui dénigre une forme d'ordre social. Néanmoins, le décalage créé par ces "nouveautés » sportives ne peut-il être appréhendé sous un angle différent de la confrontation, celui du compromis, de la médiation?

11 L'émergence de ces pratiques, dont le "Festival International des Sports Extrêmes" $(\mathrm{FISE})^{3}$ ou les «X-Games » ${ }^{4}$ signent l'exacte représentation, s'inscrit entre l'associatif et l'entrepreunarial, entre l'institution et une volonté de pratique libre, entre le sport et le spectacle, entre le professionnalisme et l'amateurisme, etc. Ce phénomène ne pourrait-il pas être observé dans la conciliation de ces systèmes de valeurs qui concentrent ces pôles? Par ce biais, en tentant de repérer les processus opérants et dynamiques, une lecture différente de la réalité sportive et sociale en jeu pourrait être le résultat d'une recherche à mener ultérieurement. En s'appuyant sur l'analyse des joutes languedociennes qui, a priori, ne semblent pas être la dernière roue du carrosse mais au contraire à l'avant garde, il s'agirait d'établir sous quelle forme un syncrétisme axiologique procède dans ces disciplines émergentes sans pour autant énoncer d'hypothèses préalables. La justesse de l'implication que propose l'observation participante, associée à la description ethnographique sur un terrain réduit, pourrait fonder les bases d'une ethnologie portée sur une activité physique en expansion dont l'intérêt a déjà suscité un travail d'enquête (Pruneau J., De Léséleuc E., Pigeassou C., 2000). 
l'attente d'une implication dans ce nouveau terrain d'enquête, l'analyse menée sur les joutes languedociennes ouvre un front de bataille qu'il faut semble-t-il mener pour accepter l'émergence d'une pensée "ternaire», source de nouveauté dans la compréhension des phénomènes actuels et notamment sportifs. Il s'agit d'essayer de comprendre les enjeux du champ sportif qui débordent largement la seule pratique traditionnelle des joutes en changeant de regard. Partout où les confrontations se cristallisent à l'aune d'une rencontre entre deux systèmes de valeurs, l'intérêt se constitue dans un regard médiateur basé sur un mode ternaire. A l'heure d'une mondialisation globalisante autant qu'une multiplication des échanges, une transformation des relations ou des identités, les rapports eux-mêmes multipliés doivent s'entendre et s'appréhender sur l'ouverture d'une autre dimension pas forcément recensée initialement. En cela, le sport ne doit plus se comprendre comme une sphère homogène mais doit se déchiffrer dans une complexité qui laisse libre court au syncrétisme, siège d'un avenir, certes, incertain, mais continu.

\section{BIBLIOGRAPHIE}

Abou S. (1981) L'Identité culturelle. Relations interethniques et problèmes d'acculturation, Paris, Anthropos.

Amselle, J- L. (1990) Logiques métisses. Anthropologie de l'identité en Afrique et ailleurs, Paris, La Découverte.

Audinet J., (1999) Le Temps du métissage, Paris, Editions de l'atelier.

Augé, M., (1975) Théorie des pouvoirs et idéologies. Etude de cas en basse Côte d'Ivoire, Paris, Hermann.

Balandier G., (1971) Sens et puissance, les dynamiques sociales, Paris, P.U.F.

Balandier G., (1974) Anthropo-logiques, Paris, P.U.F.

Bastide, R., (1967) Les Amériques noires : les civilisations africaines dans le nouveau monde, Paris, Payot. Bastide R. (1970) Le Prochain et le lointain, Paris, Cujas.

Bastide R. (1971) Anthropologie appliquée, Paris, Payot.

Bourdieu P. (1979) La Distinction. Critique sociale du jugement, Paris, Editions de Minuit.

Chaudenson R. (1979) Les Créoles français, Paris, Fernand Nathan.

Gurvitch G. (1968) La Vocation actuelle de la sociologie, Paris, P.U.F.

Herskovits M- J. (1958) Acculturation. The study of culture contact, Gloucester, (Mass.) : Peter Smith. Loret, A., (1996) «L'avènement d'un sport alternatif », in Le sport à l'épreuve (sous la direction de) P. Ceaux, Revue Lire et savoir, Paris, Gallimard Jeunesse, 3, 10-19.

Maffesoli M. (1996) Eloge de la raison sensible, Paris, Grasset.

Pruneau J., De Léséleuc E., Pigeassou C., (2000) « Fragments d'ethnologie du roller. A la recherche du sens pour éviter les contre-sens », in Cahiers Espaces, 66,124-133. 
Pruneau J. (2001) Les Joutes languedociennes. Ethnologie d'un «sport traditionnel », Thèse de Doctorat STAPS (Dir. Bromberger C., Pigeassou C.), Université Montpellier 1, Faculté des Sciences du Sport et de l'Education Physique, 320 p. plus annexes. Publiée sous le même titre (2004), Paris, Ed. L'Harmattan, Collection Espace et temps du sport.

Segalen M., (1994) Les Enfants d'Achille et de Nike, une ethnologie de la course ordinaire, Paris, Métailié.

\section{NOTES}

1. Il faut comprendre ici que trois temps structurels (avant tournoi, pendant tournoi et après tournoi) sont mis en évidence dans le système des joutes et qu'ils correspondent à l'architecture identitaire de cette pratique depuis son commencement.

2. E. De Léséleuc dans son étude anthropologique de l'escalade à Claret dégage clairement ces trois grilles de lecture concernant la nouveauté sportive. Voir E. De Léséleuc, Voler et donner. Ethnosociologie d'un lieu anthropologique : le site d'escalade de Claret, thèse de doctorat (sous la direction de) J. Gleyse, Université de Montpellier 1, janvier 2000.

3. Ce festival regroupe selon un concept à la fois compétitif, démonstratif, amical, amateur et spectaculaire les meilleurs pratiquants d'activités "nouvelles" sur une période de 3 jours dans différentes villes du monde. Une édition a lieu chaque année à Palavas-les-Flots, elle draine entre 250 et 350 mille personnes dans la station balnéaire lors du week-end Pascal.

4. Ces jeux sont au carrefour de la compétition et de l'amusement, ils sortent des cadres institutionnels habituels en proposant par exemple, lorsqu'ils mettent en présence les meilleurs skieurs extrêmes du monde, une descente de ski qui ressemble à un parcours du combattant et dans laquelle se jettent simultanément les concurrents préposés à la victoire.

\section{RÉSUMÉS}

A travers une approche ethnologique, associée à une ethnographie minutieuse de terrain, l'article proposé ici cherche à mettre en lumière un processus de transformation sociale qui vise à pérenniser une identité collective. Non révélé dans le champ des pratiques sportives, ce processus s'établit dans un espace "d'entre deux », entre tradition et modernité, et fait émerger, au-delà des considérations liées à ce "sport traditionnel ", une autre lecture du rapport entre sport et société.

Le temps métissé des joutes languedociennes, c'est la perception d'un mode "ternaire » qui déconstruit les schèmes classiques d'opposition dans lesquels l'ordre social s'inscrit depuis toujours sans considérer la voie du métissage comme un cadre possible de compréhension du système des sports. En proposant, à travers cette contribution, un ensemble de questions ouvertes, il s'agit également d'essayer, par quelques réponses, de comprendre ce que peut révéler cet espace de compromis inscrit dans « l'entre deux ». 
This work is based on an ethnological approach associated with an empirical ethnography in order to clarify a social process of transformation which aims at maintaining a collective identity. Whether this process doesn't clearly appear in the field of sport activities, it seems take place in a specific space of the "inter-period » between tradition and modernity. Beyond the considerations related to this " traditional sport », news understanding of the link between sport and society emerge.

The crossbreed time of the Languedocians tournaments is related to the perception of a "ternary" mode. This kind of phenomenon questions the social order that comes within the framework of traditional opposite conceptions. It supposes that the interbreeding way is not considered as a possible framework of comprehension of the sports system. By proposing, through this contribution, responses related to a whole of opened questions, it implies to understand what arises and be revealed from this space of compromise.

INDEX

Keywords : sport, water tournament, modernity, cultural half-breeding, tradition

Mots-clés : joutes, modernité, métissage

\section{AUTEUR}

\section{JÉRÔME PRUNEAU}

ACTES (Adaptation, Climat Tropical, Exercice, Société), Université des Antilles et de la Guyane. 